\title{
Bluthochdruck
}

\section{Neue Fixkombination für die Ersteinstellung bei Hypertonie}

_ Heute werden etwa 70\% der 20 Millionen Hypertoniker hierzulande therapiert. Bei ca. 50\% ist der Blutdruck kontrolliert, berichtete Prof. Roland Schmieder, Erlangen. Dennoch dürfe man nicht locker lassen: „Wir sollten anstreben, den Blutdruck von über $70 \%$ der Patienten zu kontrollieren."

Seit Kurzem ist eine neue Option für die initiale Therapie ab Hypertonie Grad 1 auf dem Markt: Die Fixkombination Perindopril/Amlodipin (Viaco$\left.\operatorname{ram}^{\circledast}\right)$. Schmieder erläuterte die Gründe, warum man gleich von Anfang an diese Fixkombination einsetzen sollte: Die Blutdrucksenkung ist rascher, die $\mathrm{Pa}$ tienten erreichen schneller die Zielwerte, die Tablettenlast und die Nebenwir- kungsrate sinken und die Compliance steigt.

Perindopril/Amlodipin wird in zwei Dosierungen angeboten: 3,5/2,5 mg und 7/5 mg. Mit einer einmal täglichen Einnahme wird eine zuverlässige 24-Stunden-Wirkung erzielt. Die Startdosis erwies sich in einer Vergleichsstudie mit 1.581 Patienten nach zwei Monaten als mindestens ebenso effektiv wie Monotherapien mit Perindopril $5 \mathrm{mg}$ oder Amlodipin 5 mg (RR-Senkung 22/14 vs. $18 / 11$ vs. $22 / 13 \mathrm{mmHg}$ ). Mit $44 \%$ vs. $33 \%$ vs. $38 \%$ erreichten mehr Patienten ihr Blutdruckziel unter 140/90 mmHg. Und weniger Patienten der Kombinationsgruppe beklagten Ödeme im Vergleich zu Amlodipin 5 mg (1,6\% vs. 4,9\%).
Auch in zwei großen Endpunktstudien hat sich die Kombination ACEHemmer/Kalziumantagonist anderen Kombinationen als überlegen erwiesen [ASCOT-Studie vs. Atenolol/Thiazid, Dahlof B et al. Lancet. 2005; ACCOMPLISH-Studie vs. ACE-Hemmer plus Diuretikum, Jamerson $\mathrm{K}$ et al. $\mathrm{N}$ Engl J Med. 2008]. Wesentlich sei auch die schnelle Blutdrucksenkung: Mehrere Studien haben laut Schmieder gezeigt, dass eine raschere Zielwerterreichung mit einer günstigeren Prognose einhergeht.

\section{Dr. Dirk Einecke}

- Pressegespräch „Viacoram ${ }^{\bullet}$ - neues First-Line-Therapiekonzept bei arterieller Hypertonie" im Rahmen der Herbsttagung der Deutschen Gesellschaft für Kardiologie (DGK); Berlin, Oktober 2015 (Veranstalter: Servier)

\section{Unkomplizierte Zystitis}

\section{Effektive, antibiotikafreie Doppelstrategie}

—Zystitiden sind „die häufigsten ambulant erworbenen Infektionen überhaupt", berichtete Prof. Florian Thalhammer, Wien. Bundesweit erkrankt jede zweite Frau mindestens einmal im Leben daran. Bei den meisten kommt es zu Rezidiven, so Thalhammer. In etwa $80 \%$ der Fälle seien uropathogene E.-coli-Bakterien (UPEC) die Ursache. Entsprechend sind Harnwegsinfekte laut Prof. Thalhammer auch die Hauptindikationen für Antibiotika und die Hauptursachen für die Resistenzentwicklung dagegen: „Damit ist eine Zystitis heute zur medizinischen Herausforderung geworden“"

\section{Antibiotikatherapie vermeiden}

Inzwsichen wird vor allem bei unkomplizierten Zystitiden gefordert, auf Antibiotika zu verzichten. Mit Utipro ${ }^{\circledR}$ plus kann diese erfüllt werden. Das Medizinpro- dukt enthält die drei Inhaltsstoffe Xyloglucan-Gelatine (Biofilm), Hibiskus und Propolis, die einen dualen Wirkansatz besitzen: Sie entfalten in Blase und Darm physikalische Effekte. Die XyloglucanGelatine legt sich als Biofilm über die Darmmukosa, was Anhaftung und Vermehrung von UPEC behindert und so die häufigsten Erreger reduziert. Organische Säuren aus Hibiskus und Propolis senken den $\mathrm{pH}$-Wert des Harns. Es wird vermehrt Stickstoffmonoxid gebildet, was die Bakterienproliferation hemmt.

Die Doppelstrategie bewirkt nach fünf Behandlungstagen eine signifikante Verbesserung der Symptome sowie eine Verminderung der Schmerzen beim Wasserlassen. Das Präparat ist seit September 2015 zur Therapie und Kontrolle von akuten sowie wiederkehrenden unkomplizierten Zystitiden auf dem deut-

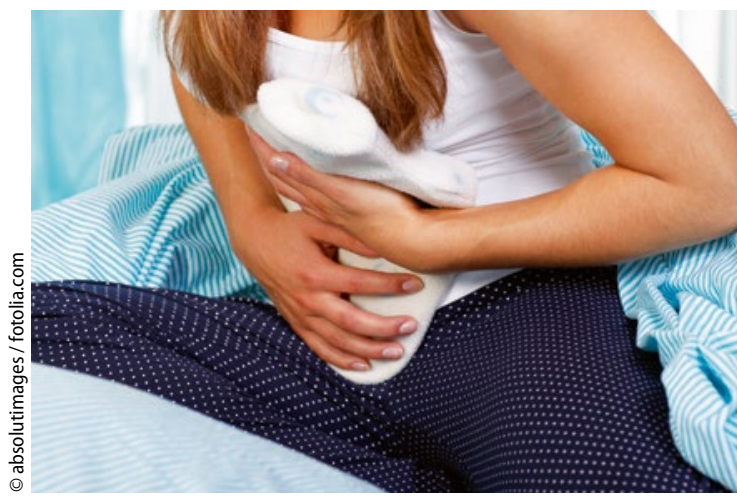

Menschen mit harmlosen Blasenentzündungen brauchen kein Antibiotikum!

schen Markt. Im akuten Fall ist die zweimal tägliche Einnahme über fünf Tage, bei Rezidiven und zur Prophylaxe die einmal tägliche Einnahme über 15 Tage pro Monat empfohlen.

Birgit Frohn

- Launch-Pressegespräch „Utipro ${ }^{\oplus}$ plus: Neuer dualer Wirkansatz zur Behandlung der unkomplizierten Zystitis"; Hamburg, September 2015 (Veranstalter: Trommsdorff) 